\title{
Planar Open Rectangle-of-Influence Drawings with Non-aligned Frames
}

\author{
Soroush Alamdari and Therese Biedl \\ David R. Cheriton School of Computer Science, University of Waterloo \\ $\{$ s26hosse, biedl\}@uwaterloo.ca
}

\begin{abstract}
A straight-line drawing of a graph is an open weak rectangleof-influence (RI) drawing, if there is no vertex in the relative interior of the axis-parallel rectangle induced by the end points of each edge. No algorithm is known to test whether a graph has a planar open weak RI-drawing, not even for inner triangulated graphs.

In this paper, we study RI-drawings that must have a non-aligned frame, i.e., the graph obtained from removing the interior of every filled triangle is drawn such that no two vertices have the same coordinate. We give a polynomial algorithm to test whether an inner triangulated graph has a planar open weak RI-drawing with non-aligned frame.
\end{abstract}

\section{Background}

The rectangle-of-influence (RI for short) drawability problem was introduced by Liotta et al. 8]. In a strong RI drawing of a graph, there is an edge between two vertices of the graph if and only if there is no other vertex in the axisparallel rectangle defined by the two ends of every edge. There are two variants of RI-drawings: In a closed RI-drawing, the rectangle required to be empty is closed, whereas in an open RI-drawing, only the relative interior of the rectangle is required to be empty.

Biedl et al. 3] introduced the concept of weak $R I$ drawings in which graphs are drawn such that for any edge the rectangle is empty, but not for all empty rectangles the edge is necessarily present. They proved that a plane graph has a planar weak closed RI drawing if and only if it has no filled triangle (i.e., a triangle that has vertices in its interior.) Furthermore, they presented an algorithm to find such a drawing in an $(n-1) \times(n-1)$ grid in linear time. The grid size can be improved to $(n-3) \times(n-3)[12$.

For open RI drawings, better bounds are known. Miura and Nishizeki [1] presented an algorithm to find a small weak open RI drawing of a given 4connected graph. Their grid size is $W \times H$ where $W+H \leq n$. Zhang and Vaidya [15] also provided small weak open RI drawings for inner triangulated 4-connected graphs with quadrangular outer face. They do this by proving that the drawing presented by Fusy [4] is a weak open RI drawing.

However, as opposed to (weak planar) closed RI-drawings of planar graphs, no necessary and sufficient conditions or testing algorithms are known for the 
existence of (weak planar) open RI-drawings, even for inner triangulated graphs. This study was initiated by Miura, Matsuno and Nishizeki [10]. They first gave necessary and sufficient conditions for planar weak open RI-drawability of triangulated planar graphs. Here all faces including the outer-face are triangles, so the outer-face is a filled triangle, which severely restricts the placement of interior vertices and facilitates testing the existence of a weak open RI-drawing.

Miura et al. 10 also aimed to develop necessary and sufficient condition for all inner triangulated graphs, but did not succeed. It is clear that such a drawing imposes conditions on how filled triangles are drawn; a natural first step is hence to remove the interior of all filled triangles and try to draw the resulting frame graph while satisfying these conditions. Miura et al. then changed their model a bit and only considered what they called oblique drawings where no edges of the frame graph are drawn horizontally or vertically. They gave one set of conditions that are clearly necessary, and showed that adding one condition made them sufficient. (See later for more details.)

In this paper, we use a slight variant of oblique drawings that we call drawings with non-aligned frame, which means that no two vertices of the frame graph have the same $x$-coordinate or the same $y$-coordinate. We give necessary and sufficient conditions for a graph to have a planar weak open RI-drawing with nonaligned frames. Our proof is algorithmic and yields a test whether a graph has a planar weak open RI-drawing with non-aligned frame; it also constructs such a drawing if one exists. Also, the algorithm works via a detour into rectangular drawings and proves a correspondence between RI-drawings and rectangular drawings that may be of independent interest.

Due to space limitations, some details have been omitted; a full version can be found in [1].

\section{Preliminaries}

Let $G=(V, E)$ be a graph with $n$ vertices $V$ and $m$ edges $E$. The graph $G$ is called simple if it has no loops or multiple edge. It is called planar if it can be drawn in the plane without crossing. A planar drawing of $G$ can be specified by giving for each vertex the cyclic order of edges around it. A planar drawing divides the plane into regions called faces. The unbounded region is called the outer face, all other faces are called inner faces. Any vertex not on the outer face is called an inner vertex. A plane graph is a planar graph with a planar embedding and the outer face specified. An inner triangulated graph is a plane graph in which every inner face is a triangle; it is called triangulated if the outer face is also a triangle. In this paper, all graphs are assumed to be simple, plane and inner triangulated, and we occasionally omit these quantifiers.

In a plane graph, a triangle is called filled if there is at least one vertex inside the triangle. Crucial for our study is the frame graph, which is the graph obtained by removing the inside of every filled triangle (see Fig. 2). Also crucial is the concept of angles of a plane graph. Each instance of a vertex appearing in a face is called an angle. The angles on the outer face are outer angles and the angles on the inner faces are called inner angles. 
Given a plane graph, the dual graph is obtained by creating a vertex $v_{f}$ for every face $f$, and adding an edge $\left(v_{f}, v_{g}\right)$ whenever faces $f$ and $g$ share an edge. The angles in the dual graph are in natural 1-1 correspondence with the angles of the original graph: The angle at vertex $v$ in face $f$ corresponds in the dual graph to the angle at vertex $v_{f}$ in the face formed where $v$ used to be.

A planar straight-line drawing of a planar graph is a drawing without crossing where all edges are straight-line segments. Such a drawing is called a planar weak open rectangle-of-influence (RI for short) drawing if for every edge $(v, w)$, the relative interior of the axis-parallel rectangle defined by the $v$ and $w$ contains no other vertex. The drawing in Fig. 1(a) is a planar weak open RI drawing. Since we do not consider any other type of RI-drawing, we omit the classifiers "planar", "weak" and "open" occasionally.
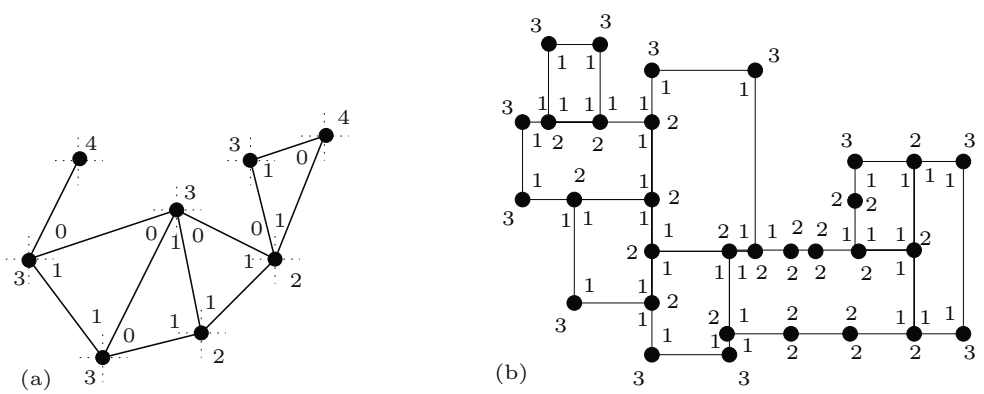

Fig. 1. (a) An oblique RI-drawing with RI-labels. (b) A rectangular drawing with corresponding RD-labels in the interior.

A straight-line drawing of a graph is oblique if no edge in the drawing is axisparallel. It is non-aligned if no axis-parallel line intersects two or more vertices of the graph. Every non-aligned drawing is oblique, but not vice versa. An inner triangulated graph has a non-aligned RI-drawing if and only if it has no filled triangle, since a non-aligned RI-drawing has no vertices on the boundaries of rectangles and hence is a closed RI-drawing.

An oblique drawing of a graph $G$ naturally induces a labeling of the angles with $\{0,1,2,3,4\}$ by assigning to each angle the number of coordinate axes contained in the angle. Since we use this concept only for RI-drawings, we call it an RI-labeling. The following is known.

Lemma 1. [10] In an oblique RI-drawing of an inner triangulated graph, the RI-labels of any inner face consists of two 1 s and one 0.

An inner triangulated graph $G$ is said to have a inner rectangular dual drawing if $G$ can be represented as the touching graph of a set of interior-disjoint axisaligned rectangles such that their union is simply connected (i.e., has no holes.) Fig. 1(b) shows an inner rectangular dual drawing of the graph in Fig. 1(a) (ignore the circles on the lines.) A rectangular dual drawing is an inner rectangular dual drawing where the union of the rectangles is also a rectangle. 
A graph has an inner rectangular dual drawing if and only if it does not have a filled triangle [14/7/6. Recall that a graph has a non-aligned RI-drawing if and only if it has no filled triangle, which suggests a relationship between these two types of drawings. We prove this formally in this paper, arguing via a third, closely related, type of drawing. A drawing of a plane graph is called an inner rectangular drawing if every edge is drawn as a horizontal or vertical line segment so that every inner face boundary is a rectangle. A rectangular drawing is an inner rectangular drawing in which the outer face is a rectangle too. See Fig. 1(b) and 5 for examples. Note that any (inner) rectangular dual drawing of a graph $G$ is an (inner) rectangular drawing of a graph that is the dual graph of $G$ except for some changes near the outer-face.

An inner rectangular drawing of a graph $G$ induces a labeling of the (graphtheoretic) angles that we call an RD-labeling: If the angle is drawn with (geometric) angle $i \pi / 2$, then assign it label $i \in\{1,2,3,4\}$. Such a labeling can be used to characterize graphs that have a rectangular drawing. Call an RD-labeling $a d$ missable if (a) each inner angle is labeled 1 or 2, (b) each inner face has exactly 4 angles of label 1, (c) for each vertex, the labels of incident angles sum to 4 , and (d) the sum of the labels on the outer-face is $2 k+4$, where $k$ is the number of angles on the outer-face.

Lemma 2. [9] A plane graph has an inner rectangular drawing if and only if it has an admissible RD-labeling.

\section{Results}

Let $G$ be an inner triangulated graph. Let $F$ be the frame graph of $G$. In this section we give a constructive algorithm to decide whether $G$ admits an open RI drawing such that $F$ is non-aligned.

Overview: Like the result by Miura et al. [10], our algorithm is based on testing whether the frame-graph $F$ of $G$ has an RI-labeling that satisfies certain restrictions, and if so, compute an RI-drawing from it. We hence review their approach first and explain the changes with our algorithm.

Miura et al. first test for every filled triangle $T$ whether the graph inside $T$ has an RI-drawing. If this fails for any $T$ then clearly $G$ has no RI-drawing either. So in the following we always assume that all interiors of all filled triangles of $T$ have an RI-drawing, at least under some restrictions on the drawing of $T$. Next, Miura et al. compute the restrictions made by a filled triangle $T$.

Lemma 3. [10] If $T=\{a, b, c\}$ is a triangle of the frame graph that is a filled triangle in $G$, and if $a$ is not adjacent to all vertices inside $T$, then in any open $R I$-drawing of $G$ with oblique frame, the induced oblique RI-drawing of the frame has RI-label 1 at a.

So there is a set $A$ of inner angles of the frame graph $F$ that must be labeled 1 in any non-aligned (hence oblique) RI-drawing of $F$ induced by an RI-drawing of $G$. Moreover, if we can find a non-aligned RI-drawing of $F$ that has these RI-labels, then it can be expanded into an open RI-drawing of graph $G$. 

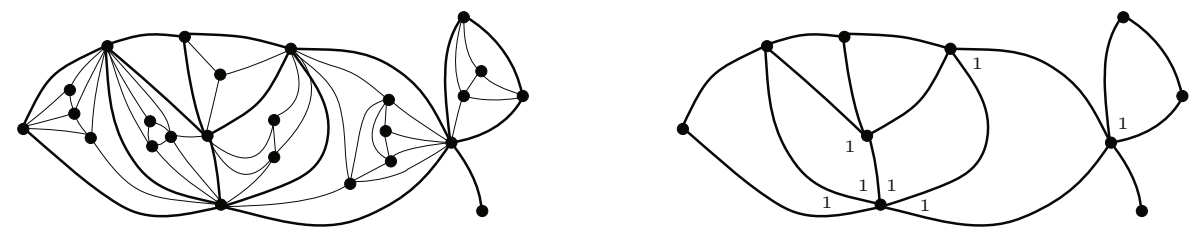

Fig. 2. Graph $G$ (left) and its frame graph $F$ with forced RI-labels (right)

Definition 1. (based on [10]) A labeling of the angles of the frame graph $F$ with $\{0,1,2,3,4\}$ is a decent RI-labeling if (a) the labels at every vertex sum to 4 , and (b) every inner triangle has labels $\{0,1,1\}$, and every angle in $A$ is labeled 1, where $A$ is the set of restriction implied from the filled triangles.

$A$ decent RI-labeling is called good if (c) the outer angles have labels $\{2,3,4\}$.

$A$ decent RI-labeling is called admissable if ( $\left.c^{\prime}\right)$ the sequence of labels on the outer angles does not contain $01^{*} 0$ as a subsequence.

Miura et al. showed that if $G$ has an open RI-drawing with oblique frame, then $F$ has a decent RI-labeling. However, they also showed a graph where this is not sufficient. Hence they added condition (c) which forces the outer-face to consist of four chains that are monotone in $x$ and $y$. This condition is not necessary, but they show that adding it gives sufficient conditions: any graph that has a good RI-labeling has an oblique RI-drawing.

We show here that using the restriction (c') gives conditions that are both necessary and sufficient, at least for the closely related concept of drawings with non-aligned frame.

Theorem 1. An inner triangulated graph $G$ has a planar weak open RI-drawing with non-aligned frame if and only if the frame graph $F$ has an admissible RIlabeling.

To prove this theorem, first consider necessity. Miura et al. already showed that conditions (a) and (b) of an admissable RI-labeling are necessary. We only sketch the proof of the necessity of condition (c'). Assume in a (planar, oblique) RIdrawing the sequence of RI-labels on the outer angles contain 00 as a subsequence, say at vertices $v_{1}$ and $v_{2}$ and edges $e_{0}, e_{1}, e_{2}$. Edge $e_{1}$ is not axis-aligned so the axis-aligned rectangle defined by its endpoints is non-trivial and must not contain the other endpoints of $e_{0}$ and $e_{2}$. But then the RI-labels of 0 force $e_{0}$ and $e_{2}$ to cross each other. Hence no (planar, oblique) RI-drawing can exist. The proof for $01^{+} 0$ as a subsequence is similar but more intricate; it is vital for this proof that the RI-drawing is non-aligned. See [1] for details.

We do not prove sufficiency directly; instead we give an algorithm that tests whether an inner triangulated $G$ has a planar weak open RI-drawing with nonaligned frame, and the steps of the algorithm imply sufficiency of an admissable RI-labeling. We outline here our algorithm: 
(i) Compute the frame graph $F$ (see Fig. 2).

(ii) For every triangle $T$ of $F$ that was filled in $G$, compute whether the interior of $T$ is realizable in an open RI-drawing [10. If this fails for any triangle, then $G$ has no open RI-drawing. Else, let $A$ be the set of inner angles of $F$ that must have RI-label 1 (Lemma 3.) See Fig. 2 .

(iii) Construct $D$ (see Fig. 3), which is roughly the dual graph of $F$ after adding one vertex in the outer-face.

(iv) Find an admissible RD-labeling of $D$ that respects $A$ in some sense. See Fig. 5] If there is none, stop: $F$ does not have a non-aligned RI-drawing (as we will show in Lemma 4.). Otherwise, convert the RD-labeling to an inner rectangular drawing by Lemma 2

(v) Expand the inner rectangular drawing $\Gamma_{D}$ into a rectangular drawing $\Gamma_{D^{\prime}}$ of a super-graph $D^{\prime}$, by adding more rectangular faces in the outside. $\Gamma_{D^{\prime}}$ also respect $A$ (see Fig. 5).

(vi) Construct the dual graph of $D^{\prime}$ and then remove the outer face vertex. The resulting graph $F^{\prime}$ is a super-graph of the frame-graph $F$ (see Fig. 6).

(vii) From the RD-labeling of $D^{\prime}$, extract an RI-labeling of $F^{\prime}$. This RI-labeling is decent, but in fact, it is good. See Fig. 6.

(viii) Using this good RI-labeling, create a non-aligned RI-drawing of $F^{\prime}$ using a variant of the algorithm presented in [10. See Fig. 7

(ix) Then insert the filled triangles (which is possible by choice of $A$ ) to obtain an open RI-drawing with non-aligned frame of a super-graph $G^{\prime}$ of $G$.

(x) Remove the vertices of $V_{G^{\prime}} \backslash V_{G}$ from the drawing (see Fig. (7).

Steps (i), (ii), (ix) and (x) are either taken from [10 or are straightforward. We give definitions and details for the other steps below.

Definition of $D$ : We first clarify how graph $D$ is defined. Let $F$ be the framegraph, i.e., $F$ is an inner triangulated graph without any filled triangle. Let $F^{+}$ be the graph obtained from $F$ by adding one vertex $v_{o}$ in its outer-face. For every outer angle $\alpha$ at a vertex $v$, we add three edges from $v$ to $v_{o}$ in $F^{+}$at the place (in the cyclic order around $v$ ) where $\alpha$ was. Thus, a vertex that appears on the outer-face of $F$ twice would have 6 edges to $v_{o}$, though not all of them would be consecutive. Now let $D$ be the dual graph of $F^{+}$. See Fig. 3 ,

Recall that there is a 1-1-correspondence between angles in a planar graph and its dual. So for every inner angle $\alpha$ of $F$ there is a corresponding inner angle $\beta$ of $D$. For every outer angle $\alpha_{i}$ of $F$, there are four corresponding inner angles $\beta_{i}^{1}, \beta_{i}^{2}, \beta_{i}^{3}, \beta_{i}^{4}$ of $D$ at the duals of the three edges from the vertex at $\alpha_{i}$ to the added vertex $v_{o}$ in $F^{+}$. See Fig. 3 .

From Admissable RI-Labelings to Rectangular Drawings: Recall that we assume the existence of a set $A$ of inner labels of $F$ that must be labeled 1 in any decent RI-labeling. We use the same set $A$ to restrict rectangular drawings of $D$. More precisely, we say that a rectangular drawing $\Gamma_{R D}$ of $D$ respects $A$ if for every angle $\alpha \in A$ (which is an inner angle of $F$ ), the corresponding angle in $\Gamma_{R D}$ has RD-label 1.

In this part, we aim to show that step (iv) is correct: If $D$ does not have a rectangular drawing that respects $A$, then $F$ does not have a non-aligned open 


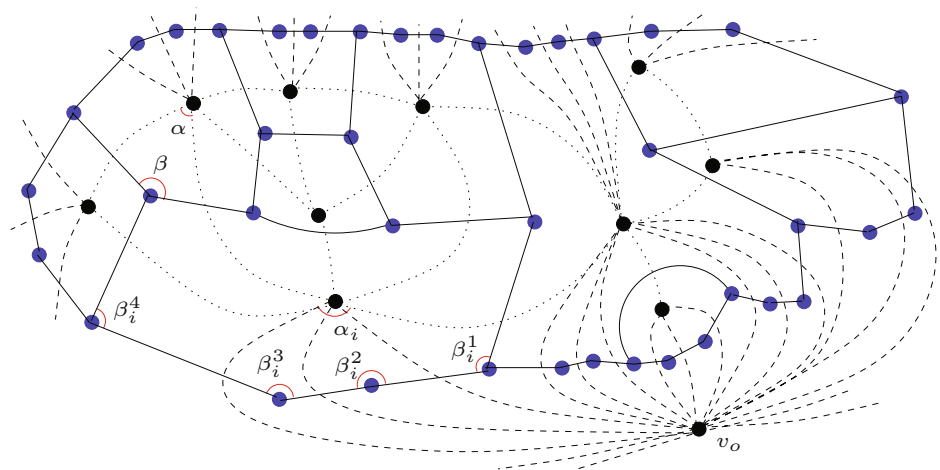

Fig. 3. The graph $F$ from Fig. 2 (dotted), the added vertex $v_{o}$ (dashed) and the graph $D$ (solid)

RI-drawing. We prove this by showing that any non-aligned open RI-drawing of $F$ can be converted to a rectangular drawing of $D$ with corresponding angles.

Definition 2. We say that an RI-labeling $\ell_{R I}$ of $F$ and an $R D$-labeling $\ell_{R D}$ of $D$ have the same inner structure if for any two corresponding inner angles $\alpha$ and $\beta$ of $F$ and $D, \ell_{R I}(\alpha)=1$ if and only angle $\ell_{R D}(\beta)=1$.

Lemma 4. For any admissible RI-labeling $\ell_{R I}$ of $F$, there exists an admissible $R D$-labeling $\ell_{R D}$ of $D$ that has the same inner structure.

Proof. Given $\ell_{R I}($.$) we define \ell_{R D}($.$) as follows: If \alpha$ is an inner angle of $F$ with corresponding inner angle $\beta$ of $D$, then set $\ell_{R D}(\beta)=2-\ell_{R I}(\alpha)$. Since $\alpha$ has label 0 or 1 , hence $\beta$ has label 1 or 2 , and it has label 1 if and only if $\alpha$ has label 1 , so the two sets of labels have the same inner structure.

If $\alpha$ is an outer angle of $F$, then assigning labels to its corresponding 4 angles of $D$ is more complicated (and in particular, not always a local operation.) Let $\alpha_{0}, \ldots, \alpha_{k-1}$ be the outer angles of $F$ in clockwise order; addition in the following is modulo $k$. For each $\alpha_{i}$, let $\beta_{i}^{1}, \ldots, \beta_{i}^{4}$ be the four corresponding inner angles of $D$, in clockwise order around the face. Now for each $i$ (see also Fig. (4):

- If $\ell_{R I}\left(\alpha_{i}\right)=0$, then assign labels $2,2,2,2$ to $\beta_{i}^{1}, \beta_{i}^{2}, \beta_{i}^{3}, \beta_{i}^{4}$.

- If $\ell_{R I}\left(\alpha_{i}\right)=2$, then assign labels $1,2,2,1$ to $\beta_{i}^{1}, \beta_{i}^{2}, \beta_{i}^{3}, \beta_{i}^{4}$.

- If $\ell_{R I}\left(\alpha_{i}\right)=3$, then assign labels $1,1,2,1$ to $\beta_{i}^{1}, \beta_{i}^{2}, \beta_{i}^{3}, \beta_{i}^{4}$.

- If $\ell_{R I}\left(\alpha_{i}\right)=4$, then assign labels $1,1,1,1$ to $\beta_{i}^{1}, \beta_{i}^{2}, \beta_{i}^{3}, \beta_{i}^{4}$.

- If $\ell_{R I}\left(\alpha_{i}\right)=1$, then we assign $1,2,2,2$ or $2,2,2,1$ to $\beta_{i}^{1}, \beta_{i}^{2}, \beta_{i}^{3}, \beta_{i}^{4}$, but the choice between these depends on the neighborhood.

Explore from angle $\alpha_{i}$ both clockwise and counter-clockwise along the outerface until we obtain a maximal subsequence where all RI-labels are 1. Say this sequence is $\alpha_{j}, \ldots, \alpha_{l}$. Since the RI-labeling is admissable, by condition (c') the sequence $\alpha_{j-1}, \alpha_{j}, \ldots, \alpha_{l}, \alpha_{l+1}$ does not have the form $01^{+} 0$, so 
one of $\alpha_{j-1}$ and $\alpha_{l+1}$ has label $\geq 2$. If $\ell_{R I}\left(\alpha_{j-1}\right) \geq 2$, then assign labels $1,2,2,2$ to $\beta_{i}^{1}, \beta_{i}^{2}, \beta_{i}^{3}, \beta_{i}^{4}$ (and also to all other corresponding angles in that subsequence), else assign labels $2,2,2,1$ to $\beta_{i}^{1}, \beta_{i}^{2}, \beta_{i}^{3}, \beta_{i}^{4}$.

Finally, for all outer angles of $D$, we set the RD-label such that the sum of labels around the vertex is 4 . We verify that the labeling is admissible:

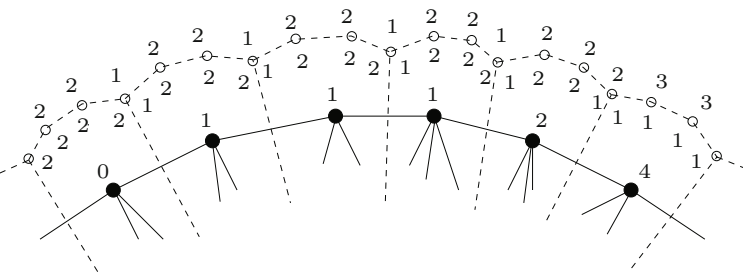

Fig. 4. Conversion of RI-labels of $F$ to RD-labels of $D$

- Each inner angle of $D$ has RD-label 1 or 2 since RI-label of inner angles of $F$ are 0 or 1 .

- Every inner face $f$ of $D$ has exactly 4 angles that have RD-label 1 . For $f$ corresponds to some vertex $v_{f}$ of $F$, and the RI-labels at $v_{f}$ sum to 4 . By construction, an RI-label $i$ at vertex $v_{f}$ gives rise to $i$ angles with RD-label 1 at $f$ (this holds even if $v_{f}$ is an outer vertex of $F$.)

- The RD-labels at every vertex $v$ of $D$ sum to 4 . For if $v$ is an inner vertex, then it corresponds to a triangle $T$ of $F$ which had RI-labels $\{0,1,1\}$, which correspond to RD-labels $\{2,1,1\}$. If $v$ is an outer vertex, then by construction of the RD-labels at outer angles of $D$ the total is 4 .

- We claim that every outer angle $\alpha$ of $D$ has RD-label $\{1,2,3\}$. Recall that $\ell_{R D}(\alpha)$ is defined as 4 minus the sum of other labels at the vertex $v$ that supports $\alpha$. Since there is at least one other label at $v$, and it is 1 or 2 , hence $\ell_{R D}(\alpha) \leq 3$.

Assume for contradiction that $\ell_{R D}(\alpha) \leq 0$. Since there are at most two inner angles at $v$, hence there must be exactly two (say $\beta_{i}^{4}$ and $\beta_{i+1}^{1}$ ) and they must both have RD-label 2. From the construction, this can happen only if $\ell_{R I}\left(\alpha_{i}\right)=0=\ell_{R I}\left(\alpha_{i+1}\right)$. But an admissable RI-labeling does not have consecutive labels 0 on the outer-face by (c'), so this cannot happen.

- Finally we must show that the number of labels on the outer face sum to $2 k+4$, where $k$ is the number of angles on the outer-face of $D$. This is a simple (but lengthy) counting-argument, which we omit for brevity's sake.

Hence the RD-labeling is admissible as desired.

Remark 1. Note that Lemma 2 implies a correspondence between inner rectangular drawings and RI-drawings: Any non-aligned RI-drawing defines an admissable RI-labeling, which implies an admissable RD-labeling, which implies an inner rectangular drawing, and they all have the same inner structure. The other direction also holds, and is proved implicitly with our algorithm. 
The contrapositive of Lemma 4 proves correctness of step (iv). If $D$ does not have an admissable RD-labeling that respects $A$, then $F$ cannot have a non-aligned RI-drawing with all angles in $A$ having RI-label 1. Fig. 5 shows an admissable RD-labeling for the restrictions of Fig. 3, and the corresponding inner rectangular drawing.
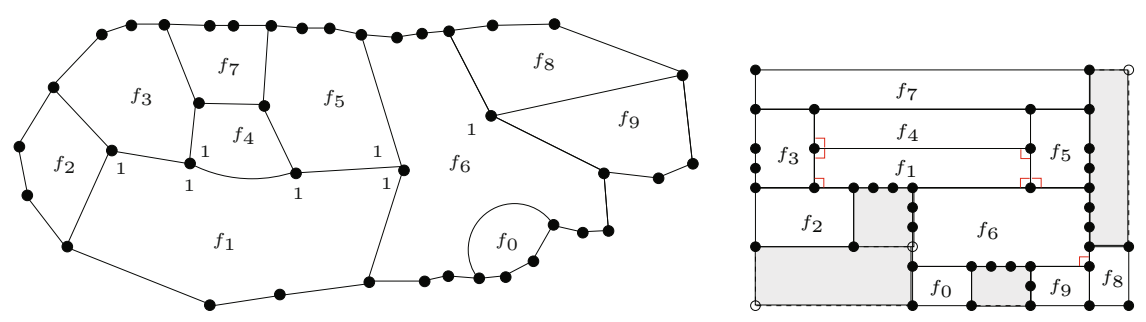

Fig. 5. Graph $D$ with the restrictions on RD-labels (left), and an inner rectangular drawing expanded to a rectangular drawing by adding shaded rectangles (right)

\section{From Inner Rectangular Drawing to Non-aligned RI-Drawing}

Lemma 5. Any inner rectangular drawing $\Gamma_{D}$ of $D$ that respects $A$ can be expanded into a rectangular drawing $\Gamma_{D^{\prime}}$ of a graph $D^{\prime}$ of size $O(|D|)$ such that inner angles of $D$ are inner angles of $D^{\prime}$, and $\Gamma_{D^{\prime}}$ respects $A$.

Proof. As part of his orthogonal-shape approach to orthogonal graph drawing, Tamassia ([13, see also [2]) provided an algorithm to add a linear number of vertices and edges to an orthogonal drawing to turn it into a rectangular drawing without changing directions of edges. The algorithm does not create any vertex of degree 4. Applying this algorithm to the inner rectangular drawing $\Gamma_{D}$ gives a rectangular drawing $\Gamma_{D^{\prime}}$ of a graph $D^{\prime}$ and only adds vertices and edges in the outer-face, since all inner faces are rectangles already. Hence all inner angles (and their RD-labels) are preserved.

Lemma 6. If $D^{\prime}$ has a rectangular drawing $\Gamma_{D^{\prime}}$ that respects $A$, then there is a super graph $F^{\prime}$ of $F$ that has a good RI-labeling.

Proof. We prove this by converting the RD-labeling of $\Gamma_{D^{\prime}}$ into an RI-labeling of $F^{\prime}$, hence more or less the reverse of the proof of Lemma 4 . Let $F^{\prime}$ be the dual of $D^{\prime}$ minus the outer face vertex. For every angle $\alpha$ of $F^{\prime}$, let $i$ be the number of angles in $D^{\prime}$ that correspond to $\alpha$ and that have RD-label 1 (i.e., their geometric angle is $\pi / 2$.) Set $\ell_{R I}(\alpha)=i$. See Fig. 6 .

Since every inner vertex of $D^{\prime}$ has RD-labels $\{1,1,2\}$ at its angles, every inner triangle of $F^{\prime}$ receives RI-labels $\{1,1,0\}$. Since every face of the RD-drawing is a rectangle, the RI-labels at any vertex of $F^{\prime}$ sum to 4 . Also, any angle in $A$ obtains RI-label 1 since its corresponding label had RD-label 1, so the resulting RI-labeling is decent. But in fact it is good: in a rectangular drawing (where the outer-face is a rectangle), any rectangle adjacent to the outer-face has at least 


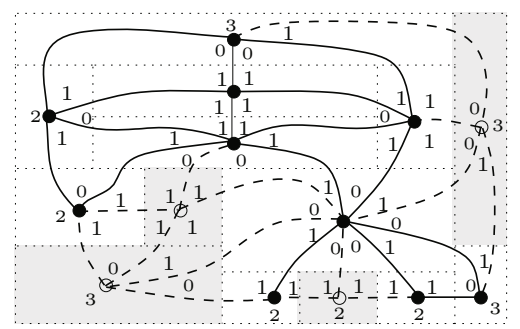

Fig. 6. The drawing $\Gamma_{D^{\prime}}$ (dotted edges) and the graph $F^{\prime}$ (left) and the corresponding good RI-labeling of $F^{\prime}$ (right)

two angles of value $\pi / 2$ on the outer-face, and so any outer angle of $F^{\prime}$ receives RI-label 2,3 or 4 .

Lemma 7. If $F^{\prime}$ has a good RI-labeling, then $F^{\prime}$ has a non-aligned RI-drawing with this RI-labeling.

Proof. We can apply Miura et al.'s algorithm to construct an RI-drawing. However, their algorithm only promises an oblique drawing; it need not be nonaligned. But we can modify their algorithm to make the drawing non-aligned. Briefly, they can show that valid coordinates can be found by solving a system of constraints. All constraints have the form of an acyclic digraph where edgeweights express lower bounds on the differences of $x$-coordinates. Since there are no upper bounds on relative $x$-coordinates, we can find a solution to this system of constraints where all $x$-coordinates are distinct (e.g. by adding edges to turn the digraph into a total order (a complete acyclic digraph) and enforcing a minimum weight of 1 on all edges.) Similarly we can compute distinct $y$-coordinates. Hence we obtained a non-aligned RI-drawing with the same RI-labels.

Putting It All Together: If a graph $G$ has an open RI-drawing with nonaligned frame $F$, then it has an admissable RI-labeling, hence $D$ has an admissable RD-labeling (Lemma 4), hence $D$ has an inner rectangular drawing (Lemma 2). and it respects $A$. Expand the inner rectangular drawing to a rectangular drawing (Lemma 5), extract a good RI-labeling from it (Lemma 6), and create a non-aligned RI-drawing from it (Lemma 7). See also Fig. 7), Insert the filled triangles and delete the added vertices and edges then results in the desired open RI-drawing with non-aligned frame of $G$. This proves correctness of the algorithm.

Our proof was constructive and gives rise to an algorithm to test whether $G$ has an open RI-drawing with non-aligned frame. It remains to analyze the run-time of this algorithm. Most steps are clearly doable in linear time. The bottleneck is the time to test whether $D$ has an RD-labeling that respects $A$.

We do this with a flow-approach inspired by Tamassia [13. We only sketch the details here. Tamassia created a flow network of a plane graph that encodes 

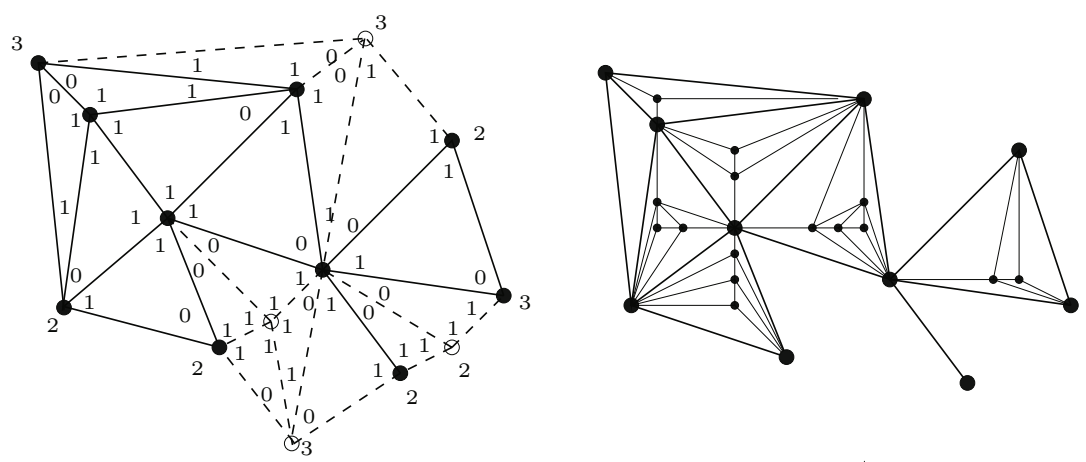

Fig. 7. The RI-drawing of $G$ obtained by the algorithm

the shapes (i.e., abstract descriptions via bends and angles) of all possible plane orthogonal drawings. It is easy to add upper and lower bounds that forbid bends on the edges, forbids reflex angles at interior faces, and forces angles in $A$ to have geometric angle $\pi / 2$. The feasible integral flows in this network then correspond to the desired RD-labels. Tamassia's result required finding a minimum-cost flow, but since we forbid bends on edges, we only need to find a feasible flow, which can be done in $O\left(n^{1.5} \log n\right)$ time [5].

Theorem 2. Let $G$ be a plane inner triangulated graph. In $O\left(n^{1.5} \log n\right)$ time, we can test whether $G$ has a planar weak open RI-drawing with non-aligned frame, and if so, construct it.

We briefly return to the suffiency for Theorem 1, If $F$ has an admissable RIlabeling, then as mentioned after Lemma 4, $D$ has a rectangular drawing that respects $A$. Steps (iv-x) of the algorithm then construct a planar weak open RI-drawing of $G$ with non-aligned frame, proving Theorem 1 .

\section{Conclusion}

We presented an algorithm to find an open RI drawing with non-aligned frame of a given inner triangulated graph $G$, if there exists such a drawing. We also characterized existence of such drawings in terms of properties of RI-labelings.

Our results also imply a correspondence between non-aligned RI-drawings and inner rectangular drawings. Lemma 4 shows that any non-aligned RI-drawing can be converted to an inner rectangular drawing with the same inner structure. Steps (iv)-(x) of our algorithm show that any inner rectangular drawing can be converted to a non-aligned RI-drawing, that preserves the inner structure. So apart from modifications near the outer-face (rectangles can "slide outward"), there is a 1-1-correspondence between non-aligned RI-drawings and inner rectangular drawings.

The most pressing open problem is what happens when we want to drop "with non-aligned frame". Can we efficiently test whether a given inner triangulated 
graph has a weak open RI-drawing? We note here that the concept of RI-labeling can be generalized quite easily to the case when the drawing is not necessarily non-aligned, if we add labels in $\{0,1\}$ to each edge with an edge labeled 1 if it is parallel to a coordinate axis. It is quite easy to find necessary conditions for such a labeling, but are they sufficient? And if they are sufficient, how easy is it to test whether a graph has a labeling that satisfies these conditions? Neither of these questions appears straight-forward to answer.

Secondly, what is the situation for planar graph that are not inner triangulated? How quickly can we test whether they have a weak open RI-drawing (perhaps under some restrictions on the frame graph)?

\section{References}

1. Alamdari, S., Biedl, T.: Planar Open Rectangle-of-Influence Drawings with NonAligned Frames. Technical Report CS-2011-17, David R. Cheriton School of Computer Science, University of Waterloo (2011)

2. Battista, G.D., Eades, P., Tamassia, R., Tollis, I.G.: Graph Drawing: Algorithms for the Visualization of Graphs. Prentice-Hall (1998)

3. Biedl, T.C., Bretscher, A., Meijer, H.: Rectangle of Influence Drawings of Graphs without Filled 3-Cycles. In: Kratochvíl, J. (ed.) GD 1999. LNCS, vol. 1731, pp. 359-368. Springer, Heidelberg (1999)

4. Fusy, E.: Transversal structures on triangulations: A combinatorial study and straight-line drawings. Discrete Mathematics 309(7), 1870-1894 (2009)

5. Goldberg, A.V., Rao, S.: Beyond the flow decomposition barrier. J. ACM 45, 783797 (1998)

6. Kozminski, K., Kinnen, E.: Rectangular dual of planar graphs. Networks 5, 145-157 (1985)

7. Leinwand, S.M., Lai, Y.-T.: An algorithm for building rectangular floor-plans. In: 21st Design Automation Conference, pp. 663-664. IEEE Press (1984)

8. Liotta, G., Lubiw, A., Meijer, H., Whitesides, S.H.: The rectangle of influence drawability problem. Computational Geometry 10(1), 1-22 (1998)

9. Miura, K., Haga, H., Nishizeki, T.: Inner rectangular drawings of plane graphs. Int. J. Comput. Geometry Appl. 16(2-3), 249-270 (2006)

10. Miura, K., Matsuno, T., Nishizeki, T.: Open rectangle-of-influence drawings of inner triangulated plane graphs. Discrete \& Computational Geometry 41(4), 643670 (2009)

11. Miura, K., Nishizeki, T.: Rectangle-of-influence drawings of four-connected plane graphs. In: Asia-Pacific Symposium on Information Visualization (APVIS). CRPIT, vol. 45, pp. 75-80 (2005)

12. Sadasivam, S., Zhang, H.: Closed rectangle-of-influence drawings for irreducible triangulations. Comput. Geom. Theory Appl. 44, 9-19 (2011)

13. Tamassia, R.: On embedding a graph in the grid with the minimum number of bends. SIAM J. Comput. 16, 421-444 (1987)

14. Ungar, P.: On diagrams representing maps. J. London Mathematical Society 28(3), 336-342 (1953)

15. Zhang, H., Vaidya, M.: On open rectangle-of-influence and rectangular dual drawings of plane graphs. Discrete Mathematics, Algorithms and Applications 1, 319-333 (2009) 